\title{
Invertible and non-invertible alloy Ising problems
}

\author{
C. Wolverton and Alex Zunger \\ National Renewable Energy Laboratory, Golden, CO 80401 \\ B. Schönfeld \\ Institut für Angewandte Physik, Eidgenössische Technische Hochschule Zürich, CH-8093 Zürich, Switzerland
}

(September 17, 2018)

\begin{abstract}
Physical properties of alloys are compared as computed from "direct" and "inverse" procedures. The direct procedure involves Monte Carlo simulations of a set of local density approximation (LDA)derived pair and multibody interactions $\left\{\nu_{f}\right\}$, generating short-range order (SRO), ground states, order-disorder transition temperatures, and structural energy differences. The inverse procedure involves "inverting" the SRO generated from $\left\{\nu_{f}\right\}$ via inverse-Monte-Carlo to obtain a set of pair only interactions $\left\{\tilde{\nu}_{f}\right\}$. The physical properties generated from $\left\{\tilde{\nu}_{f}\right\}$ are then compared with those from $\left\{\nu_{f}\right\}$. We find that (i) inversion of the SRO is possible (even when $\left\{\nu_{f}\right\}$ contains multibody interactions but $\left\{\tilde{\nu}_{f}\right\}$ does not) but, (ii) the resulting interactions $\left\{\tilde{\nu}_{f}\right\}$ agree with the input interactions $\left\{\nu_{f}\right\}$ only when the problem is dominated by pair interactions. Otherwise, $\left\{\tilde{\nu}_{f}\right\}$ are very different from $\left\{\nu_{f}\right\}$. (iii) The same SRO pattern can be produced by drastically different sets $\left\{\nu_{f}\right\}$. Thus, the effective interactions deduced from inverting SRO are not unique. (iv) Inverting SRO always misses configuration-independent (but composition-dependent) energies such as the volume deformation energy $G(x)$; consequently, the ensuing $\left\{\tilde{\nu}_{f}\right\}$ cannot be used to describe formation enthalpies or two-phase regions of the phase diagram, which depend on $G(x)$.
\end{abstract}

PACS numbers: $71.10 .+\mathrm{x}, 64.60 . \mathrm{Cn}, 64.70 . \mathrm{Kb}$

The physical properties of $A_{1-x} B_{x}$ alloys are usually analyzed and interpreted via "cluster expansion" models: 1 国 Each of the $N$ sites of an alloy $i=1, \ldots, N$ is labeled by a spin variable $\hat{S}_{i}=-1$ or +1 if site $i$ is occupied by an $A$ or $B$ atom, respectively. The set of spin variables $\left\{\hat{S}_{i}\right\}$ defines a configuration $\sigma$. The energy of any of the $2^{N}$ possible configurations is then written as a sum over clusters of points $\{i ; i j ; i j k ; \ldots\}$ :

$$
\begin{aligned}
E(\sigma, V)=J_{0}(V) & +\sum_{i} J_{i}(V) \hat{S}_{i}+\sum_{j<i} J_{i j}(V) \hat{S}_{i} \hat{S}_{j} \\
& +\sum_{k<j<i} J_{i j k}(V) \hat{S}_{i} \hat{S}_{j} \hat{S}_{k}+\ldots
\end{aligned}
$$

where $V$ is the volume, the $J$ 's are interaction energies and the first sum is over all sites, the second over all pairs, the third over all triplets, etc. We refer to these elementary clusters as "figures" $f$.

If the set of interactions $\left\{J_{f}(V)\right\}$ is known for a given alloy system, one may apply standard methods of lattice statistical mechanics (e.g., mean field, cluster variation, or Monte Carlo methods) to the expansion and compute ground state structures or finite-temperature thermodynamic properties. Recent examples include the calculation of temperature-composition phase diagrams and ground state structures of transition metall 2.3 and semiconductorla alloys, mixing enthalpies of disordered, partially ordered, and off-stoichiometric allors, short-range order (SRO) of solid solutions. 10 We refer to this approach as the "direct approach".

Conversely, another common tradition involves the "inverse approach": A measured thermodynamic prop- erty such as the set of SRO parameters $\alpha(n)$ (the atom-atom pair correlation for the $n$th atomic shell) is used in an inverse statistica approach (e.g., the inverse Monte Carlo(IMC) method ) to deduce a set of effective interactions. 3 These interactions are subsequently used in a cluster expansion [Eq. (11)] to predict thermodynamic properties other than the SRO. In this paper, we explore the extent to which the inverse approach may be used to predict alloy properties by applying it to a wellcharacterized $\alpha(n)$ obtained through a direct procedure.

In the following it is convenient to introduce the excess energy $\Delta E(\sigma, V)$ of configuration $\sigma$ defined as the energy of this configuration at volume $V$, relative to the energies $E_{A}\left(V_{A}\right)$ and $E_{B}\left(V_{B}\right)$ of equivalent amounts of solid $A$ and $B$, at their respective equilibrium volumes $V_{A}$ and $V_{B}$ :

$\Delta E(\sigma, V)=E(\sigma, V)-\left[(1-x) E_{A}\left(V_{A}\right)+x E_{B}\left(V_{B}\right)\right]$.

If the equilibrium volume $V(\sigma)$ depends primarily on the composition $x$ and only weakly on the configuration $\sigma$, then the variables $\sigma$ and $x$ can be rigorously separated in Eq. (2) giving

$$
\Delta E(\sigma, V)=G(x)+\sum_{f} D_{f} \nu_{f} \bar{\Pi}_{f}(\sigma) .
$$

Here, the first term $G(x)$ describes the elastic energy necessary to deform the constituents from their equilibrium volume to the volume $V(x)$ of $\sigma$. The second term of Eq. (3) describes the spin flip excess energy of forming $\sigma$ from $A+B$ already prepared at the volume $V$. The correlation function $\bar{\Pi}_{f}$ is defined as a product of the 
TABLE I. The values of the input interaction energies $\nu_{f}$ and the interaction energies $\tilde{\nu}_{f}$ reconstructed via IMC simulations of the SRO computed from $\nu_{f}(\mathrm{meV} /$ atom). Designation of the "figures" $f$ follows the notation of Table IV of Ref. B. For Set 2 , the multibody interactions used in the direct set $D_{f} \nu_{f}$, but not in the inverse set $D_{f} \tilde{\nu}_{f}$ are (in meV/atom): $J_{3}=-96.1$, $K_{3}=44.5, L_{3}=64.5, M_{3}=-41.1, Q_{3}=-81.3$, and $K_{4}=139.1$. Structural energy differences, ordering energies $\delta E_{\text {ord }}(\sigma)($ the energy difference between $\sigma$ and a random alloy at the same composition), and the random alloy mixing energy at $x=1 / 4$ are shown (meV/atom), as are transition temperatures $(\mathrm{K})$. "NA" means not applicable.

\begin{tabular}{|c|c|c|c|c|c|}
\hline & & \multicolumn{2}{|c|}{ Set 1} & \multicolumn{2}{|c|}{ Set 2} \\
\hline & & Direct & Inverse & Direct & Inverse \\
\hline Clusters & Designation & $D_{f} \nu_{f}$ & $D_{f} \tilde{\nu}_{f}$ & $D_{f} \nu_{f}$ & $D_{f} \tilde{\nu}_{f}$ \\
\hline Empty & $J_{0}$ & -233.2 & NA & -233.2 & NA \\
\hline Point & $J_{1}$ & 252.9 & NA & 252.9 & NA \\
\hline \multirow[t]{13}{*}{ Pairs } & $J_{2}$ & 152.0 & 157.2 & 152.0 & 690.0 \\
\hline & $K_{2}$ & -20.0 & -21.0 & -20.0 & 17.6 \\
\hline & $L_{2}$ & 58.9 & 60.0 & 58.9 & -19.2 \\
\hline & $M_{2}$ & 33.5 & 33.3 & 33.5 & 103.2 \\
\hline & $N_{2}$ & & & 0.0 & -3.6 \\
\hline & $\mathrm{O}_{2}$ & & & 0.0 & -0.4 \\
\hline & $P_{2}$ & & & 0.0 & 13.2 \\
\hline & $\delta E\left(L 1_{2}, D 0_{22}\right)$ & -4.0 & -4.8 & +103.3 & +76.2 \\
\hline & $\delta E_{\text {ord }}\left(L 1_{2}\right)$ & -42.6 & -45.1 & -41.9 & -79.8 \\
\hline & Ground State & $L 1_{2}$ & $L 1_{2}$ & $\mathrm{DO}_{22}$ & $D 0_{22}$ \\
\hline & $T_{c}$ & 630 & 680 & 1850 & 1900 \\
\hline & $G(x=1 / 4)$ & 112.1 & NA & 112.1 & NA \\
\hline & $\Delta H_{\operatorname{mix}}(1 / 4)$ & -56.2 & -172.1 & -227.7 & -600.6 \\
\hline
\end{tabular}

variables $\hat{S}_{i}$ over all sites of the figure $f$ with the overbar denoting an average over the $D_{f}$ symmetry equivalent figures per lattice site. Equation (3) is similar to Eq. (11), but here the effective interaction energies $\left\{\nu_{f}\right\}$ are volume-independent pure spin flip energies.

We will examine the invertibility of the inverse approach by performing a "controlled experiment": As input, we use two "exact" sets of interactions, $\left\{\nu_{f}\right\}$. Equation (1) is then used along with these $\left\{\nu_{f}\right\}$ in direct Monte Carlo (MC) simulations to obtain the "exact" quantities such as SRO parameters $\alpha(n)$, structural energy differences $\delta E\left(\sigma, \sigma^{\prime}\right)$ between configurations $\sigma$ and $\sigma^{\prime}$, transition temperaures $T_{c}$, and the mixing energy of the random alloy $\Delta H_{\text {mix }}$. We then contrast the results of this "direct procedure" with those of the "inverse procedure", in which the set $\{\alpha(n)\}$ (obtained in the direct procedure from the known, exact $\left.\left\{\nu_{f}\right\}\right)$ is used as input to deduce the interactions $\left\{\tilde{\nu}_{f}\right\}$ by IMC simulations from which we then obtain $\tilde{\alpha}(n), \delta \tilde{E}\left(\sigma, \sigma^{\prime}\right), \tilde{T}_{c}$, and $\Delta \tilde{H}_{\text {mix }}$.

We use as input two sets of interaction energies $\left\{\nu_{f}\right\}$ (see Table [i]). As an illustration of physically realistic interactions, we pse one set of interactions that was recently extracted 6 from $T=0$ first-principles calculations of formation energies of ordered fcc-based $\mathrm{Ni}_{1-x} \mathrm{~V}_{x}$ compounds and reproduces reasonably well many of the measured physical properties. This "realistic set", which we call Set 2, contains pair interactions up to fourth neighbors, as well as three and four-body interactions. Set 1 is identical to Set 2, except that we have set equal to zero all multibody interactions.

We first contrast the directly calculated alloy properties using Sets 1 and 2 in MC simulations. For the direct MC calculations, a system size of 4096 atoms was used with periodic boundary conditions, 1200 Monte Carlo steps (MCS) were used for equilibration, and averages were typically taken over 1800 MCS. Temperatures of $T=850 \mathrm{~K}$ and $2300 \mathrm{~K}$ were used for the SRO calculations for Sets 1 and 2. Fig. 1 shows the SRO $\alpha(\mathbf{k})$ calculated directly from $\left\{\nu_{f}\right\}$ for Sets 1 and 2 at composition $A_{3} B$. Only multibody interactions contribute to the difference between the SRO of Sets 1 and 2, and this difference is dramatic: $\alpha(\mathbf{k})$ of Set 1 shows peaks at the X-points $\langle 100\rangle$ whereas $\alpha(\mathbf{k})$ of Set 2 shows peaks at the W-points $\left\langle 1 \frac{1}{2} 0\right\rangle$ as seen experimentally in $\mathrm{Ni}_{3} \mathrm{~V}$. 10 Table il also shows that multibody interactions change the ground state structure from $L 1_{2}$ to the observed $11-D 0_{22}$ structure and that the energy difference between these two structures, $\delta E\left(L 1_{2}, D 0_{22}\right)$, changes from -4 to +103 $\mathrm{meV} /$ atom and $T_{c}$ changes from 630 to $1850 \mathrm{~K}$ upon inclusion of multibody interactions. Also, note from Table I that $G(x=1 / 4)$ is a significant fraction of the random alloy mixing enthalpy, $\Delta H_{\text {mix }}(x=1 / 4)$. Thus, from the directly calculated values it is clear that both multibody interactions and the elastic energy $G(x)$ are physically very important in this alloy system.

Using the directly calculated $\alpha(\mathbf{k})$, we now apply IMC to recover the interactions energies. Following the tradition among practitioners of the IMC method, only pair interactions were retained in the energy expression of IMC. First, configurations were produced which reproduced the input values of 35 shells of $\alpha(n)$. System sizes of 262,144 and 216,000 sites were used for Sets 1 and 2 . IMC simulations were performed on three crystals compatible with the sets of $\alpha(n)$, and averages were taken over these three crystals. Tests were performed of the convergence of the inverse procedure with respect to the 


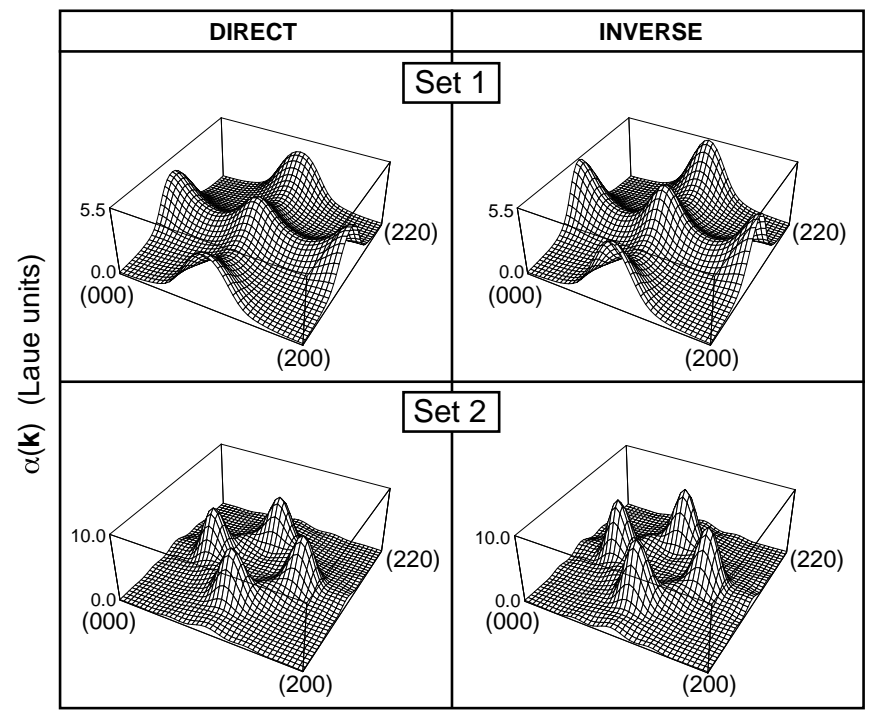

FIG. 1. Comparison of the (001) planes of $\alpha(\mathbf{k})$ and $\tilde{\alpha}(\mathbf{k})$.

number of pairs included: Many different sets of pair interactions were used (between 4 and 20 shells), and from these calculations, the number of pairs needed to adequately reproduce $\alpha(n)$ were determined.

Short-Range Order: Fig. 1 compares the recalculated $\tilde{\alpha}(\mathbf{k})$ (computed from $\tilde{\nu_{f}}$ ) and $\alpha(\mathbf{k})$ (computed from $\nu_{f}$ ). Both sets of SRO are well reproduced by the IMC procedure. The accurate inversion of SRO [i.e., $\{\tilde{\alpha}(n)\} \approx\{\alpha(n)\}]$ has been demonstrated previously by many authors (see, e.g., Refs. 7, 8). However, in these previous studies, measured SRO was used as input to the IMC, and thus the interactions which produced the input SRO were not known. We have shown that even when multibody interactions are used to produce $\alpha(n)$ (as in Set 2), the IMC procedure (using pair interactions only) reproduces this SRO quite well.

Effective Interactions: We compare the values of $\nu_{f}$ vs. $\tilde{\nu}_{f}$ in Table 1 . For Set 1, the IMC algorithm closely reproduces the input set of pair interactions; The standard deviation between $\left\{\nu_{f}\right\}$ and $\left\{\tilde{\nu}_{f}\right\}$ for Set 1 is 2.7 $\mathrm{meV} /$ atom. For Set 2, the direct and inverse sets of interactions differ dramatically in several respects: (i) Three and four body interactions are present in $\left\{\nu_{f}\right\}$, but are specifically excluded from the pair-only IMC calculation of $\left\{\tilde{\nu}_{f}\right\}$. (ii) There are huge differences in pair interactions (the standard deviation of the first four pairs is 275 $\mathrm{meV} /$ atom). (iii) Seven pair interactions were required in the IMC to reproduce $\alpha(n)$, whereas only four pair interactions were present in the direct set. (A similar increase in range was reported in Ref. 12.) However, even though there are enormous differences between $\nu_{f}$ and $\tilde{\nu}_{f}$ of Set 2, they both produce nearly identical SRO patterns (see Fig. 11). This surprising fact indicates that even the pair interactions are not determined uniquely from a SRO pattern. [This non-uniqueness was also found by Schweika and Carlsson (Ref. 12; Fig. 3a), who in contrast to the present work, used a high-temperature expansion (whereas we use IMC) to invert SRO generated by pair and multibody interactions.] We assert that due to the non-uniqueness of pair interactions derived from IMC, they cannot generally be compared with other sets of pair interactions. When multibody interactions are physically important, the non-uniqueness of these sets make such comparisons meaningless. For example, Schweika and Carlsson 12 found that inversion of SRO produced interactions $\tilde{\nu}_{f}$ which were temperature-dependent even though the input set $\left\{\nu_{f}\right\}$ was not. Clearly, this temperaturedependence is not due to physical effects (e.g., vibrational or electronic excitation effects), but rather due to the fact that a pair-only inverse scheme does not recover information on the multibody interactions $\left\{\nu_{f}\right\}$.

Structural energies, ground states, and transition temperatures computed from $\left\{\nu_{f}\right\}$ are compared with those

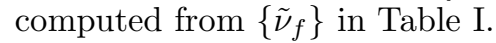

Mixing Energies: Table If also shows values of the mixing enthalpy of the random alloy $\Delta H_{\text {mix }}(x=1 / 4)$. For Set 1 , where $\left\{\tilde{\nu}_{f}\right\} \approx\left\{\nu_{f}\right\}$, the direct and inverse values of $\Delta H_{\text {mix }}$ differ by more than $100 \mathrm{meV} /$ atom, even in cases (such as Set 1) dominated by pair interactions. In Set 2 , the comparison of $\Delta H_{\text {mix }}$ is even worse (direct and inverse values differ by more than $350 \mathrm{meV}$ /atom). Deducing values of $\Delta H_{\text {mix }}$ is clearly not reliable in the inverse procedure.

We have seen that while that total energy $E(\sigma, V)$ defines the complete set of interaction energies, inversion of quantities (e.g., SRO) other than $E(\sigma, V)$ may lead to a loss of information. We now use Eq. (3) to distinguish different classes of alloy properties and discuss which are invertible:

(a) Physical properties that depend on both $G(x)$ and on the spin flip energies $\left\{\nu_{f}\right\}$ include any quantity which involves the energetics of two or more concentrations and hence, two or more volumes. [Note that $G(x)$ depends on $x$, but not on the particular atomic arrangement ("configuration") $\sigma$.] Examples include the formation energy of a structure [which involves $V, V_{A}$, and $V_{B}$, c.f. Eq. (2)], the mixing energy $\Delta H_{\text {mix }}$ of the random alloy, and two-phase equilibria in a composition-temperature phase diagram. Since "type-(a)" properties such as the set $\{\Delta E(\sigma, V)\}$ contain complete information on both $G(x)$ and on all $\left\{\nu_{f}\right\}$, given the measured or ab-initio calculated energies $\{\Delta E(\sigma, V)\}$, it is possible to invert Eq. (3) and in principle extract the "exact" $G(x)$ and $\left\{\nu_{f}\right\}$, as demonstrated in Ref. 9. Thus, inversion of "type-(a)" properties involves no loss of information.

(b) Physical properties that do not depend on $G(x)$ include energy differences of iso-compositional configurations $\sigma$ and $\sigma^{\prime}, \delta E\left(\sigma, \sigma^{\prime}\right)$. The order-disorder transition temperature $T_{c}$ at stoichiometric composition also falls into this class since it involves the energy difference between the disordered high-temperature phase and the partially ordered low-temperature phase, both at the same $x$. Another physical property which does not depend on $G(x)$ is the atomic SRO. $\alpha(n)$ involves a competition between energies of a random and a short-range ordered structure, both at the same volume $V(x)$; therefore, $\alpha(n)$ (even if determined for several compositions) 
contains no information about $G(x)$. Therefore, inversion of a "type-(b)" property, such as SRO, cannot provide any information on $G(x)$, even if the SRO covers a range of compositions. Consequently, the interactions $\left\{\tilde{\nu}_{f}\right\}$ extracted from such a procedure do not allow calculation of "type-(a)" properties, such as formation energies [Eq. (2)], mixing energies, or the phase-coexistence regions of the phase diagram. This point is highlighted by recent studies $\mathrm{B}$ on $\mathrm{Ni}_{1-x} \mathrm{Au}_{x}$ : This is a phase-separating system; however, the SRO is of ordering type. Inverting the SRO will thus inevitably produce ordering-type $\left\{\tilde{\nu}_{f}\right\}$, which are useless for predicting the miscibility gap phase diagram or the correct $\Delta H_{\text {mix }}>0$. These conflicts are resolved 5 by using $G(x)$ in the Ising-like expansion.

We conclude that: (i) The IMC algorithm provides a set of pair interactions which accurately reproduces the input SRO whether or not multibody interactions are used to generate this input. When only pair interactions are involved, the inverse procedure can even provide accurate values of structural energy differences, ordering energies, and energies of SRO; However, (ii) when multibody interactions are physically important, even the pair interactions are incorrectly determined by the inversion of SRO. The structural or ordering energies deduced from the inverse procedure can thus contain substantial errors. (iii) Finding a set of interactions which reproduces a given set of SRO is found to be a non-unique process: dramatically different sets of interactions (one set with pairs only, one set with pairs and multibodies) may still produce quantitatively the same SRO. Thus, comparing sets of interactions from IMC with other sets of interactions may be unwarranted. However, comparing a theoretical SRO pattern to a measured one is a sound procedure. (iv) Inverting the SRO always removes information on energy terms that are SRO-independent, e.g., $G(x)$. This loss prevents, in principle, the interactions deduced from SRO from being applied to predict phase-coexistence regions of the phase diagram or $\Delta H_{\text {mix }}$.

This work was supported by the Office of Energy Research (OER) [Division of Materials Science of the Office of Basic Energy Sciences (BES)], U. S. Department of Energy, under contract No. DE-AC36-83CH10093.

\footnotetext{
${ }^{1}$ F. Ducastelle, Order and Phase Stability in Alloys, Elsevier, New York (1991).

${ }^{2}$ A. Zunger, in Statics and Dynamics of Alloy Phase Transformations, P. E. A. Turchi and A. Gonis, eds., NATO ASI Series, (Plenum, New York, 1994).

${ }^{3}$ D. de Fontaine, Solid State Phys. 47, 33 (1994).

${ }^{4}$ J. M. Sanchez, F. Ducastelle, and D. Gratias, Physica A 128, 334 (1984).

${ }^{5}$ Z. -W. Lu and A. Zunger, Phys. Rev. B 50, 6626 (1994).

${ }^{6}$ C. Wolverton and A. Zunger, Phys. Rev. B 52, 8813 (1995).

${ }^{7}$ V. Gerold and J. Kern, Acta Metall. 35, 393 (1987).

${ }^{8}$ W. Schweika and H. -G. Haubold, Phys. Rev. B 37, 9240 (1988); L. Reinhard, B. Schönfeld, G. Kostorz, and W. Bührer, Phys. Rev. B 44, 1727 (1990); L. Reinhard, J. L. Robertson, S. C. Moss, G. E. Ice, P. Zschack, and C. J. Sparks, Phys. Rev. B 45, 2662 (1992).
}

${ }^{9}$ L. G. Ferreira, A. A. Mbaye, and A. Zunger, Phys. Rev. B 37, 10547 (1988).

${ }^{10}$ R. Caudron, M. Sarfati, M. Barrachin, A. Finel, F. Ducastelle, and F. Solal, J. Phys. I France 2, 1145 (1992).

11 J. F. Smith, in Phase Diagrams of Binary Vanadium Alloys (ASM International, Metals Park, OH 1987).

${ }^{12}$ W. Schweika and A. E. Carlsson, Phys. Rev. B 40, 4990 (1989). 